\title{
Sitzende Lebensweise bei Kindern und Jugendlichen
}

\author{
K. Aue ${ }^{1}$ \\ G. Huber ${ }^{2}$
}

\section{Zusammenfassung}

Lange Sitzzeiten sind ein unabhängiger Risikofaktor für die Entwicklung von Übergewicht bei Kindern und Jugendlichen. Die ansteigenden Sitzzeiten sind insbesondere auf den Medienkonsum, den motorisierten Transport und das nach wie vor sitzende Lernen in der Schule zurückzuführen. Maßnahmen gegen den Sitzenden Lebensstil sind insbesondere dann erfolgversprechend, wenn sie zielgerichtet sind und möglichst viele Faktoren einbeziehen.

Stichworte: Sitzen, Übergewicht, Risikofaktoren, Prävention
Körperliche Aktivität ist einer der zentralen Einflussfaktoren für das Wohlbefinden und die Gesundheit über die gesamte Lebensspanne. Doch parallel dazu wurde in den letzten Jahren deutlich, dass dauerhaftes Sitzen die Wahrscheinlichkeit für die Entstehung von lebensstilbedingten Erkrankungen erhöht. Zwei Reviews zeigten konsistente Befunde, dass eine sitzende Lebensweise die Entstehung von Diabetes mellitus Typ 2, kardiovaskulären Erkrankungen, Bluthochdruck und die Bildung von Gallensteinen begünstigt. Auch konnte eine Beziehung für die Inzidenz von einzelnen Krebsarten wie Ovarial-, Endometrium- und Kolon-Krebs beobachtet werden. Dabei hat das Körpergewicht bei der Krebsentstehung einen moderierenden Einfluss, während nur inkonsistente Be-

\footnotetext{
1 Plattform Ernährung und Bewegung e.V. (peb)

${ }^{2}$ Universität Heidelberg, Institut für Sport
} und Sportwissenschaft

Eingegangen: 09.03.2014

Angenommen durch Review: 16.04. 2014 funde für Übergewicht vorliegen $[8,28$, 29,44].

Für eine englische Stichprobe von Kindern (7-9 Jahre) konnte keine Zunahme des BMI durch vermehrtes Sitzen beobachtet werden [4]. Es kann dennoch davon ausgegangen werden, dass Bewegungsmangel und zunehmende sitzende Tätigkeiten energetische Dysbalancen und damit auch die Übergewichtsentstehung bereits im Kindes- und Jugendalter begünstigen [14]. So zeigte der „Global School-based Student Health Survey“ (GSHS), dass weltweit nur wenige Schüler die Empfehlungen für körperliche Aktivität und für sitzende Tätigkeiten erreichten [15]. Außerdem muss darauf verwiesen werden, dass die motorische und körperliche Leistungsfähigkeit negativ beeinflusst werden [14]. Umgekehrt stellt ein aktiver Lebensstil mit ausreichender Bewegung, d.h. mindestens moderater körperlicher Aktivität, einen Schutzfaktor für diese Krankheiten dar [17].

Daneben senkt ein Sitzender Lebensstil auch die Lebenserwartung [23]. Im Jahr 2010 hat erstmals eine Studie den Zusam- menhang zwischen Sitzzeit, körperlicher Aktivität und erhöhtem Sterberisiko untersucht. Es zeigte sich, dass Sitzzeiten von mehr als 6 Stunden pro Tag mit einer 40\% höheren Todesrate bei Frauen und einer 20\% höheren Todesrate bei Männern assoziiert sind. Dabei ist es unerheblich, ob diese Menschen sich sonst körperlich viel bewegen [27].

Das Thema Sitzen wird jedoch nicht erst im Erwachsenenalter relevant, sondern spielt bereits ab dem Krippen- und Kindergartenalter eine zentrale Rolle im Alltag. Die Bedeutung steigert sich sukzessive im Schulalltag [7,14]. Begünstigt wurde diese Entwicklung nicht zuletzt durch die Vielzahl von Hilfs- und Transportmitteln, die den Alltag erleichtern. Dazu zählen etwa Fahrzeuge, Aufzüge oder Rolltreppen [25]. Schließlich wird durch die Verhaltensweisen in Kindheit und Jugend der Grundstein für das Gesundheitsverhalten im Erwachsenenalter gelegt [12].

\section{> Sitzende Lebensweise}

Neue wissenschaftliche Erkenntnisse zeigen, dass selbst bei ausreichender Bewegung langes Sitzen einen eigenständigen und unabhängigen Risikofaktor für die Gesundheit darstellt. In der englischsprachigen Literatur wird dies als ,sedentary lifestyle“, „sedentarism“ oder „sedentary behaviour“ bezeichnet, was sich mit „sitzender Lebensweise“ oder „sitzendem Verhalten“ übersetzen lässt [8]. Eine sitzende Lebensweise schließt Verhaltensweisen ein, die mit wenig körperlicher Aktivität, einem geringem Energieaufwand oder mit Ruhe verbunden sind $[4,45]$. Sitzen 
schließt für Kinder und Jugendliche unterschiedliche Betätigungen ein [16]:

$>$ Wege zur und von der Schule mit dem Auto/ÖPNV

$>$ Lernen in der Schule (Schreiben/Lesen)

$>$ Essen und Trinken

$>$ Medienkonsum wie Fernsehen, Spielkonsolen, Computer und Smartphones in der Freizeit

Sitzen ist von einem sehr niedrigen Energieverbrauch gekennzeichnet. So liegen die energetischen „Kosten“ lediglich bei 1-1,5 MET (metabolisches Äquivalent) [8,7] (Kasten).

Somit sind sitzende Verhaltensweisen von leichter bzw. moderater (1,6-2,9 MET) und hoher, intensiverer Bewegung ( $\geq 3$ MET) abzugrenzen. Empfehlungen für körperliche Aktivität zielen auf eine moderate Intensität $\mathrm{ab}$, sodass man mindestens leicht außer Atem kommt. Für Kinder und Jugendliche wird empfohlen, dass sie sich mindestens 1 Stunde am Tag moderat bis intensiv bewegen [33]. Während körperliche Inaktivität auf dieser Empfehlung basiert, ist es möglich, dennoch übermäßig viel Zeit im Sitzen zu verbringen $[7,26,40]$. Dies konnte auch im Kindes- und Jugendalter festgestellt werden [13, 18]. Für Deutschland zeigte sich anhand des repräsentativen Kinder- und Jugendgesundheitssurveys (KiGGS), dass $46 \%$ der Jungen und $19 \%$ der Mädchen im Alter von 11-17 Jahren trotz moderater bis hoher körperlicher Aktivität die Empfehlungen für die Mediennutzung nicht einhalten konnten [3].

\section{> Prävalenzen}

Insgesamt gehen die Bewegungszeiten bei Kindern und Jugendlichen zurück, und die Sitzzeiten nehmen zu. Die von der EU geförderte HELENA-Studie zeigte, dass Ju-

\section{Metabolisches Äquivalent (MET)}

Das Metabolische Äquivalent MET (im Englischen „metabolic equivalent of task“) beschreibt den Stoffwechselumsatz eines Menschen bezogen auf den Ruheumsatz im Verhältnis zu seinem Körpergewicht. $1 \mathrm{MET}$ entspricht dem Umsatz von 3,5 ml Sauerstoff/kg Körpergewicht/Min. bei Männern, bei Frauen sind es 3,15 ml/kg Körpergewicht/ Min. Eine andere Definition bezeichnet als 1 MET einen Kalorienverbrauch von 4,2 kJ (1 kcal) je kg Körpergewicht/Stunde. Beides entspricht in etwa dem Ruheumsatz des Körpers. Moderate körperliche Aktivität hat etwa einen Energieverbrauch von 3-6 MET, intensive Anstrengungen hingegen $>6 \mathrm{MET}$ (Abb. 1).

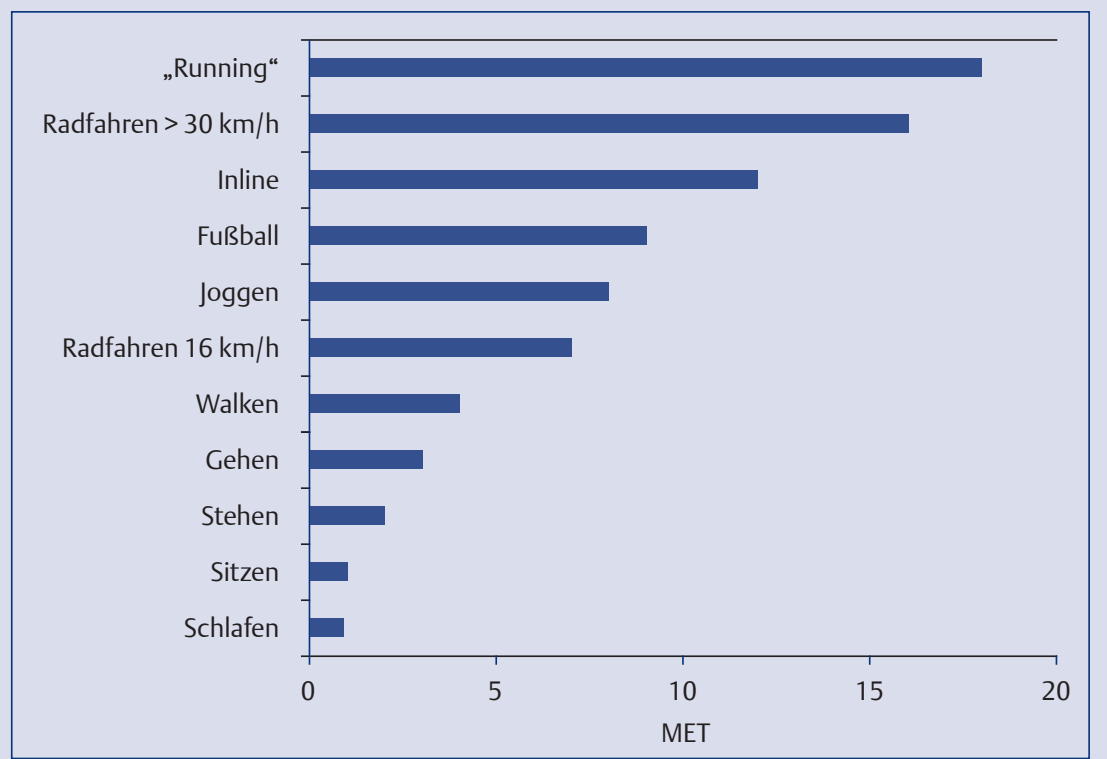

Abb. 1 Das metabolische Äquivalent - Definition und Beispiele (modifiziert nach [1]).

gendliche zwischen 12 und 17 Jahren in Europa im Durchschnitt 9 Stunden/Tag sitzen, das sind mehr als 70\% ihrer Wachzeit [34]. Der US-amerikanische National Health and Nutrition Examination Survey (NHANES) zeigt für 12-15-Jährige Sitzzeiten zwischen 7,4 Stunden (Mädchen) und 7,7 Stunden (Jungen) auf. Für 6-11Jährige lagen diese Werte bereits bei 6 Stunden/Tag und bei den 16-19-jährigen Teilnehmern bei 8 Stunden/ Tag [21].

Sitzen ist zumeist eine Ko-Aktivität, denn im Vordergrund steht eine andere eigentliche Zieltätigkeit, die eben im Sitzen ausgeführt wird. Wichtige Primäraktivitäten bei Kindern und Jugendlichen, die im Sitzen ausgeführt werden, sind Computerund TV-Konsum, der Schulweg und die Zeit in der Schule $[7,16]$. Am häufigsten üben Schüler sitzende Tätigkeiten zu Hause, in der Schule oder in der Nachbarschaft aus [43]. Verloigne et al. [47] weisen daher darauf hin, dass die Bildschirmzeit allein kein geeigneter Indikator zur Ermittlung der gesamten Sitzzeit darstellt. Nachfolgend werden einzelne relevante Bereiche vorgestellt.

\section{Bildschirmzeiten}

Obwohl die empfohlenen Bildschirmzeiten für Schüler bei max. 1-2 Stunden/Tag liegen und Bildschirmmedien aus den Kinderzimmern entfernt werden sollten [2], zeigen speziell für Deutschland die Daten der KiGGS-Studie, dass sich 11-17-Jährige im Durchschnitt zwischen 2,7 Stunden/Tag (Mädchen) und 3,8 Stunden/Tag (Jungen) mit audiovisuellen Medien beschäftigen [40]. Dazu zählen Fernsehen, Computerspiele, Social Media und Spielekonsolen. All diese sitzenden Tätigkeiten sind beliebte Freizeitbeschäftigungen von Kindern und Jugendlichen. Medienkonsum erhöht die Sitzzeiten und verdrängt somit andere aktive Beschäftigungen [31]. Zudem haben Studien ergeben, dass Kinder und Jugendliche mit längeren Bildschirmzeiten eine höhere Energieaufnahme [11] und eine ungünstige Lebensmittelauswahl aufweisen [28]. Dies kann zum einen dadurch bedingt sein, dass Mahlzeiten vor dem Fernseher eingenommen werden, Kinder dadurch unachtsamer essen und vergleichsweise spät bemerken, wann sie satt sind. Zum anderen kann das „Snacken“ vor dem Fernseher als Begründung angeführt werden. 
Die HELENA-Studie zeigte, dass 60\% der europäischen Jugendlichen am Wochenende mehr als 2 Stunden/Tag fernsehen, wobei Zeiten vor dem Computer oder der Spielkonsole noch nicht mit eingerechnet sind. Bei jungen Kindern gibt es keine Unterschiede zwischen Jungen und Mädchen in Bezug auf die Zeiten des TV-Konsums. Im Jugendalter verbringen Jungen mehr Zeit vor Computer und Fernseher als Mädchen [32]. Kinder mit niedrigerem Sozialstatus verbringen mehr Zeit vor dem Fernseher als Kinder mit höherem sozialen Status [6]. Weiterhin sind bei Kindern, die einen eigenen Fernseher im Kinderzimmer haben, lange Fernsehzeiten wahrscheinlicher $[32,39]$. Betrachtet man die vergangenen 40 Jahre, so lässt sich zusammenfassend feststellen, dass Kinder immer früher anfangen fernzusehen. So begannen Kinder 1971 den TV Konsum mit 4 Jahren, 2007 setzte der TV-Konsum bereits im Alter von 5 Monaten ein [48]. Auffällig ist, dass der Fernsehkonsum bei Kindern mit Übergewicht korreliert, während Lesen oder die nicht schulische Computernutzung keinen Zusammenhang aufweist [41].

\section{Wege zur Kita und zur Schule}

Ein immer geringerer Anteil von Kindern und Jugendlichen legt Wege zur Kita und Schule aktiv (zu Fuß, mit dem Fahrrad) zurück. So werden Kinder häufig mit dem Auto zur Schule gebracht oder fahren mit dem Bus $[22,38]$. Für Deutschland zeigt die MoMo-Studie (Motorikmodul der KiGGSStudie), dass 19,4\% der Jugendlichen zu Fuß und 22,2\% mit dem Fahrrad zur Schule kommen. Alle anderen kamen auf einem passiven Weg, d.h. mit Bahn, Bus oder Auto. Es konnte kein Zusammenhang zwischen einem aktiven Schulweg und dem relativen Körpergewicht gefunden werden. Dennoch befürworten die Autoren, dass der Schulweg aktiv bestritten wird, da auf diese Weise die Gesundheit positiv beeinflusst werden kann $[14,30]$.

\section{Schule}

Mit dem Schuleintritt steigen die Sitzzeiten von Kindern sprunghaft an. Dementsprechend gehen die Bewegungszeiten zurück. Die Zeiten, die Kinder sitzend in der Schule verbringen, sind ein wichtiger Bestandteil der gesamten Sitzzeit. Es gibt jedoch kaum Studien, die eindeutig zeigen, wie lange genau die nicht aktive Zeit von Kindern in der Schule ist und ob diese in den letzten beiden Jahrzehnten zugenommen hat [35]. Während es eine Vielzahl von Untersuchungen zur Nutzung von Bildschirmmedien gibt, ist die Datenlage für andere sitzende Aktivitäten unzureichend. Eine Untersuchung, die sich auf 9-16-Jährige konzentrierte, ermittelte, dass $60 \%$ der gesamten sitzenden Zeit den Nicht-Bildschirmmedien zuzuordnen sind. Der größte Anteil betrifft den Bereich Schule mit $42 \%$ sowie soziale Aktivitäten (Gesprächeführen) mit 19\%. Des Weiteren sind Tätigkeiten wie Essen und der passive Transport mit Auto und ÖPNV an dieser Stelle zu nennen [24].

\section{$>$ Bedingungsfaktoren für eine sitzende Lebensweise im Kindes- und Jugendalter}

Derzeit sind korrelative Zusammenhänge zwischen dem Sitzenden Lebensstil bei Kindern und Jugendlichen in unterschiedlichen Bereichen bekannt. Beispielsweise steigen die „Sitzzeiten“ mit dem Alter an. Auch gibt es Hinweise darauf, dass Jungen zwar mehr fernsehen, Mädchen dennoch häufiger sitzen. Höhere Sitzzeiten sind auch bei Personen mit niedrigem Sozialstatus und bei Personen mit Migrationshintergrund zu beobachten. Ebenso haben Eltern einen hohen Stellwert in Bezug auf die Sitzzeiten: Dazu zählen Regeln im Umgang mit Medien genauso wie das eigene Vorbild. Gleichsam ist die Anzahl von Medien, insbesondere im Kinderzimmer, ausschlaggebend. Schließlich begünstigen Sicherheitsbedenken hinsichtlich dessen, Kinder allein draußen spielen zu lassen, eine sitzende Lebensweise [7].

\section{$>$ Stand der Praxis}

Projektansätze, die gezielt auf eine Verringerung des inaktiven Verhaltens hinwirken, zielen zumeist darauf ab, die Sitzzeiten der Kinder durch verringerten TVKonsum zu verkürzen; andere adressieren den Schulweg oder Unterbrechungen der Sitzzeiten in der Schule. Die Evaluationsergebnisse dieser Projekte zeigen, dass es möglich ist, die Sitzzeiten von Kindern und Jugendlichen $\mathrm{zu}$ reduzieren $[5,46]$. Interventionen zur allgemeinen Erhöhung der körperlichen Aktivität sind erfolgreicher, wenn die Interventionen auf Theorien der Verhaltensänderung basieren [37]. In Bezug auf die Veränderung des inaktiven Verhaltens haben sich vor allem jene Theorien als erfolgreich erwiesen, die mehrere Einflussebenen auf das Gesundheitsverhalten einbeziehen wie z.B. interpersonelle und politische Faktoren oder die physische Umgebung [36]. Besonders erfolgreich sind Maßnahmen dann, wenn ausschließlich auf die Verringerung und Unterbrechung der Sitzzeiten abgezielt wird und nicht zusätzlich weitere Verhaltensänderungen das Ziel der Intervention sind [42].

\section{$>$ Was heißt das für die Praxis?}

Die Reduzierung von Sitzzeiten ist für die Gesundheitsförderung grundsätzlich sinnvoll. Bereits regelmäßige Unterbrechungen des Sitzens haben einen positiven Effekt auf die Gewichtsentwicklung und den allgemeinen Gesundheitszustand. Daher sollte das Sitzen mindestens alle 20 Min. kurz unterbrochen werden. In der Schule sollte in diesem Sinne jede Unterrichtsstunde durch mindestens ein Bewegungselement unterbrochen werden. Das stärkt zusätzlich die Konzentrations- und Leistungsfähigkeit. Die „Transform-Us-Studie“ zeigt, dass 2-minütige Unterbrechungen gut in den Schulalltag integriert werden können [36]. Lehrer und Schüler bewerten diese kurze aktive Unterbrechung positiv. $\mathrm{Zu}$ betonen ist auch, dass Bewegungseinheiten nicht nur für den Sportunterricht oder die Pause sinnvoll sind. Das Handbuch „Unterricht in Bewegung“ zeigt anhand von Beispielen für die Grundschule, wie Bewegung auch in den Deutsch-, Mathematik- und Sachunterricht sinnvoll eingebracht werden kann [9].

Tägliche Wegstrecken, z.B. zur Kita oder zur Schule, sollten, wann immer möglich, mit dem Fahrrad oder zu Fuß zurückgelegt werden. Eine erfolgreiche Initiative hierzu ist der „Walking Bus“, bei dem Schulkinder wie ein Linienbus nach Fahrplan fest vereinbarte „Haltestellen“ anlaufen, wobei sie von einem Erwachsenen begleitet werden. 
So füllt sich der „Walking Bus“ nach und nach und bringt die Kinder sicher zur Schule und nach dem Unterricht wieder nach Hause. Wie verschiedene Modellprojekte in Deutschland zeigen, fördert der „Walking Bus“ nicht nur den aktiven Schulweg, sondern begünstigt auch die Verkehrssicherheit, reduziert Umweltbelastungen und verbessert das Gemeinschaftsleben in sozial benachteiligten Quartieren [10].

Eine Studie, die die Effekte dieses laufenden Buses untersuchte, zeigte, dass Kinder von dem Fußmarsch auf dem Schulweg in Bezug auf das Ausmaß ihrer täglichen Bewegung profitieren. Darüber hinaus wurden Hinweise gefunden, dass der Schulweg zu Fuß die Konzentrationsleistung von Grundschülern über den gesamten Schulvormittag nachhaltig steigern kann [19].

Online zu finden unter
http://dx.doi.org/
10-1055/s-0034-1373870

\section{Literatur}

1 Ainsworth BE, Haskell WL, Whitt MC et al. Compendium of physical activities: an update of activity codes and MET intensities. Med Sci Sports Exerc 2000; 32: S498-504

2 American Academy of Pediatrics. Children, Adolescents, and Television. Pediatrics 2001; 107: 423-426

3 Aue K. Lebensmittelverzehr und Aktivitätsverhalten bei 11- bis 17-jährigen Jugendlichen (2008). Masterarbeit im Fach Ökotrophologie an der ChristianAlbrechts-Universität zu Kiel. Im Internet: http://edoc.rki.de/docviews/abstract. php?lang=ger\&id=363; Stand 4.3.14

4 Basterfield L, Pearce MS, Adamson AJ et al. Physical activity, sedentary behavior, and adiposity in English children. Am J Prev Med 2012; 42: 445-451

5 Biddle SJ, O'Connell S, Braithwaite RE. Sedentary behaviour interventions in young people: a meta-analysis. $\mathrm{Br}$ J Sports Med 2011; 45: 937-942

6 Brettschneider W. Study on young people's lifestyles and sedentariness and the role of sport in the context of education and as a means of restoring the balance. Final report. Paderborn: Universität Paderborn; 2004

7 Bucksch J, Dreger S. Sitzendes Verhalten als Risikofaktor im Kindes- und Jugendalter. Präv Gesundheitsf 2014; 9: 39-46
8 Bucksch J, Schlicht W [2014] Sitzende Lebensweise als ein gesundheitlich riskantes Verhalten. Dtsch Z Sportmed 2014; 65: 15-21

9 BZgA. Unterricht in Bewegung. Materialien für die Grundschule [1.-4. Klasse]. Köln; 2013

10 Conrad A, Bunge C. Walkability aus Sicht der Umweltwissenschaft. In: Bucksch J, Schneider S, Hrsg. Walkability. Das Handbuch zur Bewegungsförderung in der Kommune, Bern: Hans Huber; 2014: 105-113

11 Epstein LH, Roemmich JN, Paluch RA et al. Influence of changes in sedentary behavior on energy and macronutrient intake in youth. Am J Clin Nutr 2005; 81 : 361-366

12 Gordon-Larsen P, Nelson MC, Popkin BM. Longitudinal physical activity and sedentary behavior trends. Am J Prev Med 2004; 27: 277-283

13 Gorely T, Marshall SJ, Biddle SJ et al. Patterns of sedentary behaviour and physical activity among adolescents in the United Kingdom: Project STIL. J Behav Med 2007; 30: 521-531

14 Graf C, Beneke R, Bloch W et al. Vorschläge zur Förderung der körperlichen Aktivität von Kindern und Jugendlichen in Deutschland. Monatsschr Kinderheilkd 2013; 161: 439-446

15 Guthold R, Cowan M], Autenrieth CS et al. Physical activity and sedentary behavior among school-children: a 34-country comparison. J Pediatr 2010; 157: 43-49.e1

16 Huber G. „Generation S“. Moderne Ernährung heute 2013; Heft 1: 1-6

17 Huber G. Ist Sitzen eine tödliche Aktivität? B\&G 2014; 30: 13-16

18 Jago R, Fox KR, Page AS et al. Physical activity and sedentary behaviour typologies of 10-11 year olds. Int J Behav Nutr Phys Act 2010; 7: 59

19 Kehne M. Zur Wirkung von Alltagsaktivität auf kognitive Leistungen von Kindern. Sportforum 2011; Vol. 26. Aachen: Meyer \& Meyer; 2011

20 Lampert T, Sygusch R, Schlack R. Nutzung elektronischer Medien im Jugendalter. Bundesgesundheitsbl 2007; 50: 643-652

21 Matthews CE, Chen KY, Freedson PS et al. Amount of time spent in sedentary behaviors in the United States, 20032004. Am J Epidemiol 2008; 167: 875-881

22 McDonald NC. Active transportation to school: trends among U.S. schoolchildren, 1969-2001. Am J Prev Med 2007; 32: 509-516

23 Nelson MC, Gordon-Larsen P, Adair LS et al. Adolescent physical activity and sedentary behavior: patterning and long-term maintenance. Am J Prev Med 2005; 28: 259-266

24 Olds TS, Maher CA, Ridley K et al. Descriptive epidemiology of screen and non-screen sedentary time in adolescents: a cross sectional study. Int J Behav Nutr Phys Act 2010; 7: 92
25 Owen N, Healy GN, Matthews CE et al. Too Much Sitting. Exerc Sport Sci Rev 2010; 38: 105-113

26 Pate RR, O'Neill JR, Lobelo F. The evolving definition of „sedentary“. Exerc Sport Sci Rev 2008; 36: 173-178

27 Patel AV, Bernstein L, Deka A et al. Leisure time spent sitting in relation to total mortality in a pro-spective cohort of US adults. Am J Epidemiol 2010; 172: 419-429

28 Pearson N, Biddle SJ, Stuart JH. Sedentary behavior and dietary intake in children, adolescents, and adults. A systematic review. Am J Prev Med 2011; 41: 178-188

29 Proper KI, Singh AS, van Mechelen W et al. Sedentary behaviors and health outcomes among adults: a systematic review of prospective studies. Am J Prev Med 2011; 40: 174-182

30 Rauner A, Schmocker E, Reimers A et al. Aktiver Schulweg und relatives Körpergewicht bei Jugend-lichen in Deutschland: Ergebnisse der MoMo-Studie. In: Becker S, Hrsg. Aktiv und Gesund? Wiesbaden: Springer Fachmedien; 2014: 181-193

31 Rey-Lopez JP, Vicente-Rodriguez G, Biosca $M$ et al. Sedentary behaviour and obesity development in children and adolescents. Nutr Metab Cardiovasc Dis 2008; 18: 242 251

32 Rey-López JP, Vicente-Rodriguez G, Ortega FB et al. Sedentary patterns and media availability in European adolescents: The HELENA study. Prev Med 2010; 51: 50-55

33 RKI, BZgA. Erkennen - Bewerten Handeln: Zur Gesundheit von Kindern und Jugendlichen in Deutschland. 2008

34 Ruiz JR, Ortega FB, Martínez-Gómez D et al. Objectively measured physical activity and sedentary time in European adolescents: the HELENA study. Am J Epidemiol 2011; 174: 173-184

35 Salmon J. Novel strategies to promote children's physical activities and reduce sedentary behavior. J Phys Act Health 2010; 7 Suppl 3: S299-S306

36 Salmon J, Arundell L, Hume C et al. A cluster-randomized controlled trial to reduce sedentary be-havior and promote physical activity and health of 8-9 year olds: the Transform-Us! study. BMC Public Health 2011; 11: 759

37 Salmon J, Booth ML, Phongsavan P et al. Promoting physical activity participation among children and adolescents. Epidemiol Rev 2007; 29: 144-159

38 Salmon J, Timperio A. Prevalence, trends and environmental influences on child and youth physical activity. Med Sport Sci 2007; 50: 183-199

39 Santaliestra-Pasías AM, Mouratidou T, Verbestel $V$ et al. Physical activity and sedentary behaviour in European children: the IDEFICS study. Public Health Nutr 2013; im Druck

40 Sedentary Behaviour Research Network. Letter to the Editor: Standardized use of the terms „sed-entary“ and "sedentary 
behaviours“. Appl Physiol Nutr Metab 2012; 37: 540-542

41 Sisson SB, Broyles ST, Baker BL et al. Television, reading, and computer time: correlates of school-day leisure-time sedentary behavior and relationship with overweight in children in the U.S. J Phys Act Health 2011; 8 Suppl 2: S188-S197

42 Steeves JA, Thompson DL, Bassett DR et al. A review of different behavior modification strategies designed to reduce sedentary screen behaviors in children. J Obes 2012: 1-16

43 Taverno R, Byth W, Dowda M et al. Sedentary behaviors in fifth-grade boys and girls: where, with whom, and why? Child Obes 2013; 9: 532-539

44 Thorp AA, Owen N, Neuhaus M et al. Sedentary behaviors and subsequent health outcomes in adults a systematic review of longitudinal studies, 19962011. Am J Prev Med 2011; 41: 207-215

45 Trilk JL, Pate RR, Pfeiffer KA et al. A cluster analysis of physical activity and sedentary behavior pat-terns in middle school girls. J Adolesc Health 2012; 51: 292-298

46 van Grieken A, Ezendam N, Paulis W et al. Primary prevention of overweight in children and adolescents: a meta-analysis of the effectiveness of interventions aiming to decrease sedentary be-haviour. Int J Behav Nutr Phys Act 2012; 9: 61

47 Verloigne M, van Lippevelde W, Maes L et al. Self-reported TV and computer time do not represent accelerometer-derived total sedentary time in 10 to 12 -year-olds. Eur J Public Health 2013; 23: 30-32

48 Zimmerman FJ, Christakis DA, Meltzoff AN. Television and DVD/video viewing in children younger than 2 years. Arch Pediatr Adolesc Med 2007; 161: 473-479
Korrespondenzadresse

Dr. Katja Aue

Plattform Ernährung

und Bewegung e.V.

(peb)

Wallstraße 65

10179 Berlin

Tel. 030/278797-68

E-Mail: k.aue@pebonline.de

\section{Summary}

\section{Sedentary lifestyle in children and youth}

A long sitting period is an independent risk factor for the development of obesity in children and youth. The increase in the time spent while sitting is particularly linked to media consumption, motorized transport and of course „seated-learning“ in schools. Measures against sedentary lifestyle are especially promising, if they are objectiveoriented and involve many other relevant factors.

Key words: sitting, obesity, risk factors, prevention 